\title{
La dramaturgia de Moreto \\ en su etapa de madurez (1655-1669)
}

\author{
María Luisa Lobato \\ Universidad de Burgos \\ mlobato@ubu.es
}

Fecha de recepción: 15/10/2010, Fecha de publicación: 04/11/2010

<URL: http://www.studiaaurea.com/articulo.php?id=141>

\section{Resumen}

Se analiza la cronología de la obra dramática total de Agustín Moreto, diferenciando las etapas de producción y difusión de las obras.

\section{Palabras clave}

Agustín Moreto, teatro del Siglo de Oro, comedia, Antonio García de Prado, Diego Osorio, Sebastián de Prado, Juan de la Calle.

\section{Abstract \\ Moreto's Dramaturgy in his Late Years (1655-1669) \\ An analysis of the chronology of Agustín Moreto's entire dramatic oeuvre that distinguis- hes between the various stages in which the works were written and circulated. \\ Key words \\ Agustín Moreto, Early Modern dramaturgy, comedy, Antonio García de Prado, Diego Osorio, Sebastián de Prado, Juan de la Calle.}


Agustín Moreto ha sido reconocido por la crítica contemporánea como el dramaturgo más importante del tercer cuarto del siglo XVII, ${ }^{1}$ pero su éxito en las tablas tanto en aquella centuria como en la siguiente no llevó aparejada la dedicación del dramaturgo a la publicación de sus comedias, cuestión ésta en la que tampoco fue una excepción de su tiempo. Como en otros momentos he indicado ${ }^{2}$, sólo la Primera parte de comedias de Agustín Moreto se imprimió durante su vida y pensamos que él mismo se encargó de recopilar las doce comedias que vio oportunas. La dedicatoria de aquella Primera parte de 1654 a Francisco Fernández de la Cueva, Duque de Alburquerque, permite atisbar el deseo del dramaturgo de ofrecer un regalo al amigo y mecenas, al mismo tiempo que doce de sus comedias salían para México, ciudad de donde era virrey en aquellos momentos Fernández de la Cueva, y en la que podían encontrar así una nueva proyección. ${ }^{3}$

\section{Acerca del proceso de recopilación de la Primera parte de comedias de Agustín Moreto (1654)}

Un aspecto nuevo que nuestro equipo Proteo ha aportado a la historia de esta producción teatral es la relación que guardan varias comedias de esa Primera parte ${ }^{4}$ con circunstancias vitales por las que atravesó el mismo Fernández de la Cueva, a quien le fueron ofrecidas. Es el caso, por ejemplo, de $E l$ desdén, con el desdén, centrada en Barcelona, que podría tener al propio Duque de Alburquerque como una sombra tras el protagonista Carlos. Una segunda comedia, De fuera vendrá, contiene una extensa relación sobre el socorro de Girona,

\footnotetext{
* Esta aportación se enmarca en el proyecto FFI2010-16890 (Subprograma FILO) del MICINN dentro de la actividad del Grupo de Investigación Proteo de la Universidad de Burgos, www.ubu.es/proteo, y en el proyecto Consolider Ingenio del MICINN, ref. CSD2009-00033.
}

1. Vitse (1984): 593.

2. Lobato (2009).

3. Dice así el inicio de la dedicatoria: «Excelentísimo Señor: Consolábase Ovidio de que su libro fuese solo a la ciudad, por excusarse de la prolija censura de los críticos y del agudo diente de sus émulos, que ordinariamente se toma por agudeza el decir mal. Y considerándolo todo dijo: "Parve — nec invideo — sine me, liber, ibis in Urbem". Pero yo, que envío mi libro no donde ha de ser censurado, sino donde se ha de ver aplaudido; no donde ha de ser envidiado por los aplausos que él se pueda adquirir, sino por las honras que le ha de dar el dueño a quien va dedicado, ¿̇con qué me podré consolar?, quedando entre los embates de la murmuración y viéndole ir a lograr los frutos que no le puede negar la sombra de la grandeza de Vuestra Excelencia, a cuyo sagrado va dirigido».

4. Compuesta por los siguientes títulos: $L a$ fuerza de la ley, El mejor amigo, el rey, El desdén, con el desdén, La misma conciencia acusa, De fuera vendrá, Hasta el fin nadie es dichoso, El poder de la amistad, Trampa adelante, Antioco y Seleuco, Los jueces de Castilla, El lego del Carmen [San Franco de Sena] y Lo que puede la aprehensión. En la actualidad el grupo Proteo se ocupa de la edición crítica de esta Parte, de la que Edition Reichenberger de Kassel (Alemania) ha publicado hasta el momento los volúmenes I (2008) y IV (2010). 
como si quisiera darse noticia de lo ocurrido a Fernández de la Cueva, el cual había intervenido con eficacia en las guerras de Cataluña antes de su ida a México. Se nombra en ella, además, al propio hermano del Duque, Gaspar de la Cueva, entre quienes fueron responsables de la victoria militar. También en la comedia de esta Parte titulada Hasta el fin nadie es dichoso hay relación argumental con los intentos de secesión catalana hasta la capitulación final de Barcelona en octubre de 1652. Por otra parte, en La misma conciencia acusa, la acción se sitúa en Parma, pero Moreto traza la historia a partir de la obra de Lope El despertar a quien duerme, casi cuarenta ańos anterior y centrada también en Barcelona.

Otras cuestiones dan cierta unidad a esta recopilación, como el hecho de que buen número de las comedias de la Primera parte se las había vendido Moreto al autor Gaspar Fernández de Valdés y, tras la muerte de éste en septiembre de 1654, Moreto se las pidió a la viuda de este comediante, Damiana Arias de Peñafiel, pagando lo necesario al no haberse agotado aún el tiempo de explotación en las tablas de las obras por parte de esa compañía.

El porqué de la decisión de Moreto de reunir doce de sus comedias en 1654 puede estar relacionado, además, con el hecho de que aquel mismo año se había publicado la colección Teatro poético en doce comedias nuevas de los mejores ingenios de España. Séptima parte (Madrid, Domingo García y Morrás, 1654), que contenía tres de las obras que Moreto preparaba de forma simultánea para la publicación en su Primera parte: El poder de la amistad, La misma conciencia acusa y La fuerza de la ley. Coincidieron entre ambas colecciones: Teatro poético y Primera parte, varios de los implicados en el proceso, en concreto, quienes dieron la aprobación, la licencia del ordinario eclesiástico, la licencia de impresión, la fe de erratas y la tasa.

Con sutileza, Zugasti recoge la frase de fray Diego Niseno escrita el 2 de junio de 1654 en los preliminares de Teatro poético, en que indica que recuerda haber visto algunas de aquellas comedias: «Estas comedias [...] ya yo las tenía vistas y censuradas para otros particulares fines, y como entonces no hallé en ellas cosa alguna que se opusiese al verdadero sentir de nuestra católica fe [...], agora juzgo lo mismo». Y, en efecto, el censor las había visto aquel mismo verano en la colección preparada por Moreto, pero sólo de una forma parcial, porque dos de los textos recogidos en Teatro poético ${ }^{5}$ distaban mucho de los originales moretianos, tanto que sus editores del equipo Рвотео $^{6}$ establecen la hipótesis de que debieron ser versiones preparadas por un memorión. ${ }^{7}$

5. El poder de la amistad y La misma conciencia acusa.

6. Zugasti, en prensa, y Di Pinto para La misma conciencia acusa, en "Prólogo» a su edición de esta comedia, en prensa.
7. Sobre memoriones y el temor que los dramaturgos tenían a su tarea, véanse las opiniones de Lope de Vega recopiladas en El teatro según Lope de Vega (2009) vol. I: 179-182, entradas 110 y 111 . 
Primera parte de comedias de Agustín Moreto (1654)

\begin{tabular}{|l|l|}
\hline $\begin{array}{c}\text { Fecha aproximada de composición } \\
\text { de comedias contenidas en esa } \\
\text { recopilación }\end{array}$ & \multicolumn{1}{c|}{ Título } \\
\hline 1651 tragedia de honor conyugal & La fuerza de la ley \\
\hline a.1648 comedia de privanza & El mejor amigo, el rey \\
\hline $1651-1652$ palatina & El desdén, con el desdén \\
\hline a.1654 palatina & La misma conciencia acusa \\
\hline 1653 de capa y espada & De fuera vendrá \\
\hline a.1654 palatina & Hasta el fin nadie es dichoso \\
\hline 1652 palatina & El poder de la amistad \\
\hline 1651 de capa y espada & Trampa adelante \\
\hline a.1654 palatina seudohistórica & Antíoco y Seleuco \\
\hline 1648 seudohistórica & Los jueces de Castilla \\
\hline $1651-1652$ hagiográfica & San Franco de Sena o El lego del Carmen \\
\hline $1648-1653$ palatina & Lo que puede la aprehensión \\
\hline
\end{tabular}

\section{Otra producción de Moreto en el primer periodo (1637-1654), ajena a la Primera parte de comedias}

Examinada ya la voluntad de Moreto de recopilar en 1654 doce de sus comedias en vida y los motivos que le pudieron llevar a ello, hay que señalar que ésta fue la única Parte de comedias de la que se ocupó personalmente, pues la que aquí nos va a interesar, la Segunda parte de comedias, se imprimió en 1676, siete años tras su muerte ocurrida en octubre de 1669. Y la razón de que no recopilase comedias en una nueva Parte no fue, desde luego, que no produjera más durante ese periodo. Como ya en otro lugar indiqué, ${ }^{8}$ en el primer período de producción moretiana, el que va desde 1637 a 1654, Moreto compuso no menos de veintiún comedias en solitario, entre las que se cuentan, además de las doce consignadas en la Primera parte, otras nueve tituladas: Merecer para alcanzar o La fortuna merecida, Hacer del contrario amigo o Empezar a ser amigos, La cena del rey Baltasar, El Eneas de Dios o El caballero del sacramento o El blasón de los Moncadas, El licenciado Vidriera, La confusión de un jardín, Los siete durmientes o Los más dichosos hermanos, El parecido y El caballero.

Comenzando aún antes, en 1637, colaboró con otros dramaturgos al menos en dieciocho comedias. ${ }^{9}$ En 1637 participó en La renegada de Valladolid o La cau-

8. Lobato $(2008 a): 34-35$.

9. Para las colaboradas me apoyo en la inves- tigación de Cassol, 2008: 165-184, a la que añado nuevos datos. 


\begin{tabular}{|l|l|}
\hline \multicolumn{1}{|c|}{ Fecha composición } & \multicolumn{1}{c|}{ Título } \\
\hline 1637 & Merecer para alcanzar o La fortuna merecida (atribución dudosa) \\
\hline ca. 1638 & Hacer del contrario amigo o Empezar a ser amigos (atribución dudosa) \\
\hline a.1648 & La cena del rey Baltasar \\
\hline a.1648 & $\begin{array}{l}\text { El Eneas de Dios o El caballero del sacramento o El blasón de los } \\
\text { Moncadas (atribución dudosa) }\end{array}$ \\
\hline 1648 & El licenciado Vidriera \\
\hline a.1649 & La confusión de un jardín \\
\hline 1651 & Los siete durmientes o Los más dichosos hermanos (atribución dudosa) \\
\hline 1652 & El parecido \\
\hline 1652 & El caballero \\
\hline
\end{tabular}

*La hizo Bartolomé Romero a Su Majestad el 8 de diciembre de 1637 (Rennert, 1907-1908: 47).

tiva de Valladolid (Belmonte, Moreto y Martínez de Meneses), a la que siguieron antes de junio de 1643 La mejor luna africana (Belmonte, L. Vélez, J. Vélez, Alfaro, Moreto, Martínez de Meneses, Sigler de Huerta, Cáncer, Rosete) y de abril de 1645 El príncipe perseguido (Belmonte, Moreto, Martínez de Meneses). En 1651 se imprimió su comedia escrita con Matos: El príncipe prodigioso y también con ese dramaturgo, pero sin que por el momento podamos situarla cronológicamente, escribió El mejor par de los doce. Antes de 1652 se compuso La fingida Arcadia (Moreto, un dramaturgo menor, Calderón). ${ }^{10}$ Se editaron en julio de 1653 Oponerse a las estrellas (Matos, Martínez de Meneses y Moreto) y Nuestra Señora del Pilar (Villaviciosa, Matos y Moreto). Moreto escribió antes de la muerte de Cáncer, ocurrida el 2 de octubre de 1655, ${ }^{11}$ todas las colaboradas con él: Nuestra Señora de la Aurora (Cáncer y Moreto), La adúltera penitente (Cáncer, Moreto y Matos), El bruto de Babilonia (Matos, Moreto y Cáncer), Caer para levantar o San Gil de Portugal (Matos, Cáncer y Moreto), La fuerza del natural (Cáncer, Moreto y Matos), Hacer remedio el dolor (Moreto y Cáncer), El rey don Enrique el Enfermo (Cáncer, Zabaleta, Martínez, Rosete, Villaviciosa, Moreto) y El hijo pródigo (Moreto y Cáncer). Vida y muerte de San Cayetano (Diamante, Villaviciosa, Avellaneda, Matos, Arce, Moreto) es de otoño de 1655, según los Avisos de Barrionuevo. ${ }^{12}$

10. Véanse al respecto los trabajos de Rull (2008) y Trambaioli (2008).

11. Se incluyen en esta primera parte de su producción (1637-1654), porque aunque Cáncer murió el 2 de octubre de 1655 las obras pudieron estar escritas con cierta anterioridad. Una segunda razón es que interesa marcar como fin de esta primera etapa la fecha emblemática de 1654 en que Moreto publicó su Primera parte de comedias. 12. "Hase compuesto una comedia grande de San Gaetano, de todos los mejores ingenios de la corte, con grandes tramoyas y aparatos; y estando para hacerse, la recogió la Inquisición. No creo tienen cosa contra la fe, si bien 


\begin{tabular}{|c|c|}
\hline Fecha composición & Título \\
\hline 1637 & $\begin{array}{l}\text { La renegada de Valladolid o La cautiva de Valladolid (Belmonte, } \\
\text { Moreto y Martínez de Meneses) }\end{array}$ \\
\hline a. junio 1643 & $\begin{array}{l}\text { La mejor luna africana (Belmonte, L. Vélez, J. Vélez, Alfaro, Moreto, } \\
\text { Martínez de Meneses, Sigler de Huerta, Cáncer y Rosete) }\end{array}$ \\
\hline a. abril 1645 & El príncipe perseguido (Belmonte, Moreto, Martínez de Meneses) \\
\hline Ca. 1648 & Nuestra Señora de la Aurora (Cáncer y Moreto) \\
\hline 1648 & Oponerse a las estrellas (Matos, Martínez de Meneses y Moreto) \\
\hline 1648 & Hacer remedio el dolor (Moreto y Cáncer) \\
\hline 1651 & La adúltera penitente o Santa Teodora (Cáncer, Moreto y Matos) \\
\hline 1651 & $\begin{array}{l}\text { El principe prodigioso o Defensor de la fe o La defensa de la fe (Matos } \\
\text { y ¿Moreto?) }\end{array}$ \\
\hline 1651 & El bruto de Babilonia (Matos, Moreto y Cáncer) \\
\hline a. 1652 & La fingida Arcadia (Moreto, un dramaturgo menor y Calderón) \\
\hline a. julio 1653 & Nuestra Señora del Pilar (Villaviciosa, Matos y Moreto) \\
\hline a. 2 octubre 1655 & No hay reino como el de Dios o Mártires de Madrid (atribución dudosa) \\
\hline a. 2 octubre 1655 & Caer para levantar o San Gil de Portugal (Matos, Cáncer y Moreto) \\
\hline a. 2 octubre 1655 & La fuerza del natural (Cáncer, Moreto y Matos) \\
\hline a. 2 octubre 1655 & $\begin{array}{l}\text { El rey don Enrique el Enfermo (Cáncer, Zabaleta, Martínez, Rosete, } \\
\text { Villaviciosa y Moreto) }\end{array}$ \\
\hline a. 2 octubre 1655 & El hijo pródigo (Moreto y Cáncer) \\
\hline a. 2 octubre 1655 & $\begin{array}{l}\text { Vida y muerte de San Cayetano (Diamante, Villaviciosa, Avellaneda, } \\
\text { Matos, Arce y Moreto) }\end{array}$ \\
\hline s.a. & El mejor par de los doce (Moreto y Matos) \\
\hline
\end{tabular}

\section{Acerca del proceso de recopilación de la Segunda parte de comedias de Agustín Moreto (1676)}

De las treinta y nueve comedias citadas compuestas hasta 1655, algunas fueron recogidas en la Segunda parte de comedias (1676), veinte años después de haber sido compuestas y representadas. Hay que decir, por tanto, que este volumen de 1676 no recoge únicamente obras de la madurez creativa de Moreto, aunque también, como muestra el caso de El lindo don Diego (1662). Conviene tener

lo apócrifo debe de ser mucho. La Reina se muere por verla y las mujeres dicen locuras. Paréceme que, en viniendo el Rey, se represen- tará, según dicen. Tanto es el afecto del pueblo y género femenino» (Barrionuevo, 1968, vol. I: 212). 
en cuenta que casi la mitad de las obras de esta colectánea fueron escritas en el primer periodo de su producción, al que hemos datado entre 1637-1654.

La descripción de la princeps en el ejemplar que hemos consultado es la siguiente:

Segunda Parte de las comedias de don Agustín Moreto [1ª edición]. Dedicadas al ilvstre señor don Francisco Ydiaquez, Butrón y Múxica, Borja, Marques de San Damian, \&c. [grabado con escudo] Pliegos 64. Con licencia, en Valencia, en la Imprenta de Benito Macè, junto al Real Colegio del Señor Patriarca. Año de 1676. A costa de Francisco Duart [sic], Mercader de libros. [Portada ilustrada con un precioso escudo. Sin colofón. FIN. Sigue un grabado [jarrón con flores]. [Con errores en la paginación]. No puede ser, pp. 1-44; Santa Rosa, pp. 45-88; La Fuerza del Natural, pp. 89-124; Primera es la honra, pp. 125-164; El Licenciado Bidriera, pp. 165204; Industrias contra Finezas, pp. 205-244; El Cavallero. pp. 256-289; El Parecido, pp. 291-334; La Fingida Arcadia, pp. 335-370; El Cavallero del Sacramento, pp. 371-418; El Valiente Justiciero, pp. 419-458; El Lindo Don Diego, pp. 459-496. Biblioteca del Institut del Teatre de Barcelona, 59211 ejemplar completo y en buen estado. Portada restaurada. Encuadernado en chagrín de color azul, con florones en el lomo. Procedencia: Cotarelo.

El editor fue Benito Macè, el cual desarrolló su trabajo junto al Colegio del señor Patriarca ${ }^{13}$ de Valencia entre 1660-1676, y a su muerte ocurrida en enero de $1677^{14}$ la imprenta pasó a su viuda, Josefa Avinent, y en 1691 a sus herederos. El fallecimiento de Benito Macé ocurrió, pues, poco después de la impresión de la Segunda parte de comedias de Agustín Moreto, que habría reunido en fechas cercanas a la impresión. También aquel último año de su vida, 1676, salió de sus prensas la Comedia sin música de Andrés Dávila y Heredia, sátira en prosa de las representaciones musicales. Pero la mayoría de las obras que imprimió fueron sermones y obras religiosas.

Esta Segunda parte la costeó Francisco Duarte, mercader de libros, con quien Macé ya había colaborado en ocasiones anteriores, por ejemplo en 1675, en la edición de la Gramática de Nebrija comentada por Antonio Cerezo. ${ }^{15}$ Desconocemos con exactitud las razones que llevaron a Duarte a querer reunir y dedicar este volumen de Moreto a Francisco Idiáquez Butrón Mújica Borja, Marqués de San Damián, e hijo de quien tenía — entre otros títulos- el de gentilhombre de la Cámara de Su Majestad, Montero y Ballestero Mayor del rey Felipe IV y Sumiller de Corps del príncipe Baltasar Carlos. Lo que resulta más significativo y se puede añadir a lo anterior es que el padre del dedicatario era Capitán General de Valencia en aquellos momentos.

13. Se trataba del Real Colegio Seminario del Corpus Christi, fundado en 1586 por el Patriarca de Antioquía San Juan de Ribera, que fue arzobispo y virrey de Valencia. El edificio se terminó en 1615.

14. Serrano (1898-1899): 261-262.

15. Nebrisensis (1675). 
Segunda parte de comedias de Agustín Moreto (1676)

\begin{tabular}{|l|l|}
\hline $\begin{array}{c}\text { Fecha aproximada composición de } \\
\text { comedias contenidas en esa } \\
\text { recopilación }\end{array}$ & \multicolumn{1}{|c|}{ Título } \\
\hline a.1648 apologética & $\begin{array}{l}\text { El Eneas de Dios o El caballero del sacramento o El } \\
\text { blasón de los Moncadas }\end{array}$ \\
\hline 1648 palatina & El licenciado Vidriera \\
\hline a.1652 palatina & $\begin{array}{l}\text { La fingida Arcadia (Moreto, un dramaturgo menor, } \\
\text { Calderón) }\end{array}$ \\
\hline 1652 capa y espada & El parecido \\
\hline 1652 capa y espada & El caballero \\
\hline a. 2 octubre 1655 palatina & La fuerza del natural (Cáncer, Morato y Matos) \\
\hline 1657 capa y espada & El valiente justiciero o El ricohombre de Alcalá \\
\hline 1659 capa y espada & No puede ser el guardar una mujer \\
\hline 1662 palatina & Primero es la honra \\
\hline 1662 de figurón & El lindo don Diego \\
\hline 1666 palatina & Industrias contra finezas \\
\hline ca. 1669 hagiográfica & Santa Rosa del Perú (Moreto y Lanini) \\
\hline
\end{tabular}

Interesa también recordar el pasaje de la dedicatoria firmada en Valencia el 27 de noviembre de 1675 en que Francisco Duarte se refiere a la producción de Moreto:

Ofrezco a los pies de V.S.I. la Segunda parte de Comedias de Don Agustin Moreto, para que con su patrocinio pueda sublimarse a la cumbre del aplauso. Dos cosas he conseguido en la impresión de este libro: la primera, la elección de comedias, por ser de cómico tan aplaudido y con justa razón celebrado. La segunda, el acierto en ampararme de la sombra de V.S.I. para que tenga todo el complemento de Grande en la corte de la Fama, y si la nobleza está obligada a patrocinar cualquier desvalido, como lo siente Casaneo, ${ }^{16}$ esta vez le coge de lleno a V.S.I. el empeño, pues tan circuido le veo de ella. Y así es obligación en V.S.I. conceder el valimiento que procuro, por las razones que verá en este periodo.

El resto de la dedicatoria es una laudatio de la familia de su protector. En la cita anterior dedicada a Moreto, Francisco Duarte alaba al dramaturgo indicando de él que es "cómico tan aplaudido y con justa razón celebrado", y

16. Catalogus gloriae mundi, p. 8. cons. 19, es la nota que aparece en la dedicatoria. Es una cita de la obra de Casaneo (1529). 
parece hacerse responsable de la elección de las doce comedias que conforman el volumen.

Puede tener interés, por tanto, observar cuáles son los géneros que Duarte eligió para esta colectánea dedicada al hijo del virrey de Valencia, entre los que se encuentran, principalmente, comedias de capa y espada y palatinas. Entre las primeras están El parecido, El caballero, El valiente justiciero o El ricohombre de Alcalá y No puede ser el guardar una mujer. Son palatinas, género que Moreto practicó con asiduidad: El licenciado Vidriera, La fingida Arcadia, La fuerza del natural, Primero es la honra e Industrias contra finezas. A ellas se suman tres obras de signo distinto: un ejemplo de comedia de figurón: El lindo don Diego, una apologética: El Eneas de Dios y una hagiográfica: Santa Rosa del Perú. De entre ellas, nueve están escritas en solitario mientras que La fingida Arcadia y La fuerza del natural las escribió en colaboración con otros dramaturgos y Santa Rosa la terminó Lanini tras la muerte de Moreto.

La aprobación de la Segunda parte es de Tomás López de los Ríos, quien conocía la obra dramática impresa de Moreto, como indica su referencia a ella:

Estas comedias de don Agustín Moreto corren ya impresas y aplaudidas en diferentes tomos; en las de éste, cuya impresión se pretende repetir en Valencia, no puedo añadir aprobación sino continuar la que tantos hombres doctos han hecho de unas y otras comedias del mismo autor, y con mucha razón, porque cuantas ha querido escribir las ha sabido acertar con gala, con propiedad, con ejemplo y con admiración.

Las contenidas en este tomo la merecen y la conseguirán, y más autorizadas con la licencia que desea quien las ha juntado y que, a mi sentir, se puede conceder por no hallarse cláusula que se oponga a la verdad y pureza de la fe ni al decoro y piedad de las buenas costumbres. Así lo siento y firmo en Valencia a 20 de febrero de 1676.

Reconoce, por tanto, López de los Ríos que esas comedias están ya impresas, la mayoría de ellas — podemos añadir — en las diversas colecciones de Escogidas, ${ }^{17}$ aunque habría que indicar que no tenemos constancia de dónde se

17. Las partes de "Comedias escogidas» que contienen comedias de Moreto son las tituladas: Primera parte. Comedias escogidas de los mejores ingenios de España, Madrid, Domingo García y Morrás, a costa de Juan de San Vicente, 1652, BNE, R/22654.- Quinta parte. Comedias escogidas de los mejores ingenios de España, Madrid, Pablo de Val, a costa de Juan de San Vicente, 1653, BNE, R/22658. - Séptima parte. Teatro poético en doce comedias nuevas de los mejores ingenios de España, Madrid, Domingo García y Morrás, a costa de Domingo de Palacios, 1654, BNE, R/22660.- Parte nona. Come- dias escogidas de los mejores ingenios de España, Madrid, Gregorio Rodríguez, a costa de Mateo de la Bastida, 1657, BNE, R/22662.- Parte décima. Nuevo teatro de comedias varias de diferentes autores, Madrid, Imprenta Real, a costa de Francisco Serrano de Figueroa, 1658, BNE, R/22663.- Oncena parte. Comedias nuevas escogidas de los mejores ingenios de España, Madrid, Gregorio Rodríguez, a costa de Iuan de San Vicente, 1659, BNE, R/22664.- Parte duodécima. Comedias nuevas escogidas de los mejores ingenios de España, Madrid, por Andrés García de la Iglesia, a costa de Iuan de San Vi- 
imprimió El parecido, del que nos consta, sin embargo, que estaba escrito desde 1652. En cuanto a la aprobación del volumen, poco tiene que añadir a lo que ya han señalado sus antecesores en los volúmenes de Comedias Escogidas y en otros. En todo caso, valga destacar su apreciación de que Moreto acertó sus comedias "con gala, con propiedad, con ejemplo y con admiración», para indicar — de forma ya más estereotipada — que concede la aprobación «por no hallarse cláusula que se oponga a la verdad y pureza de la fe ni al decoro y piedad de las buenas costumbres».

El citado Tomás López de los Ríos era bien conocido en la zona de Valencia. Había actuado como fiscal en diversas Academias barrocas valencianas, por ejemplo en la que tuvo lugar en el convento de la Encarnación en 1665 y, más cerca de la fecha que aquí interesa, había terminado la obra empezada por Baltasar Sopena y Zarzuela, que se interrumpió por la muerte de éste, y que Jerónimo

cente, 1658, BITB, 58432-44.- Parte catorce. Pensil de Apolo en doze comedias nueuas de los meiores ingenios de España, Madrid, Domingo García Morras, a costa de Domingo Palacio y Villegas, 1661, BNE, R/22667.- - Parte quince. Comedias nuevas, escogidas de los mejores ingenios de España, Madrid, Melchor Sánchez, a costa de Juan de San Vicente, 1661, BNE, R/22668. - Parte diez y siete. Comedias nuevas, y escogidas de los mejores ingenios de Europa, Madrid, Melchor Sánchez, a costa de Juan de San Vicente, 1662, BNE, R/ 22670.-Parte diez y ocho, Comedias nuevas, escogidas de los mejores ingenios de España, Madrid, Gregorio Rodríguez, y a su costa, 1662, BNE, R/22671.Parte diez y nueve. Comedias nuevas, y escogidas de los mejores ingenios de España, Madrid, Pablo de Val, a costa de Domingo Palacio y Villegas, 1663, BNE, R/22672._- Parte veinte y quatro. Comedias nuevas y escogidas de los mejores ingenios de España, Madrid, por Mateo Fernández de Espinosa Arteaga, a costa de Iuan de San Vicente, 1666, BNE, R/ 22677._- Parte veinte y cinco de comedias nuevas, y escogidas de los mejores ingenios de España, Madrid, Domingo García Morrás, a costa de Domingo Palacio y Villegas, 1666, BNE, R/22678._- Parte veinte y nueve. Comedias nuevas, escritas por los mejores ingenios de España, Madrid, Ioseph Fernández de Buendía, a costa de Manuel Meléndez, 1668, BNE, R/ 22682.- Parte treinta. Comedias nuevas, y escogidas de los mejores ingenios de España, Madrid, Domingo García Morrás, a costa de Domingo Palacio y Villegas, 1668, BNE, R/ 22683.- Parte treinta y cuatro. Co- medias nuevas escritas por los mejores ingenios de España, Madrid, Joseph Fernández de Buendía, a costa de Manuel Meléndez, 1670, BNE, R/ 22687. - Parte treinta y cinco. Comedias nuevas, escritas por los mejores ingenios de España, Madrid, Lucas Antonio de Bedmar, a costa de Antonio de la Fuente, 1671, BNE, R/ 22688.Parte treinta y seis. Comedias nuevas, escritas por los mejores ingenios de España, Madrid, Ioseph Fernández de Buendía, a costa de Manuel Meléndez, 1671, BNE, R/ 22689._- Parte treinta $y$ siete. Comedias nuevas escritas por los mejores ingenios de España, Madrid, Melchor Alegre, a costa de Domingo Palacio y Villegas, 1671, BNE, R/ 22690. - Parte treinta y nueve de Comedias nuevas escritas por los mejores ingenios de España, Madrid, Joseph Fernández de Buendía, a costa de Domingo de Palacio y Villegas, 1673, BNE, R/ 22692. - Parte quarenta y una de famosas comedias de diferentes autores, Pamplona, José del Espíritu Santo, 1675, BNE, R/ 22694 (impresa, al parecer, en dos partes). Cotarelo dice que esta portada es falsa y que la impresión es de Madrid, y probablemente de 1675 (CCPBE).- Parte quarenta y dos. Comedias nuevas, nunca impressas, escogidas de los mejores ingenios de España, Madrid, Roque Rico de Miranda, a costa de Iuan Martín Merinero, 1676, BNE, R/ 22695. - Parte cuarenta y tres. Comedias nuevas de los mejores ingenios de España, Madrid, Antonio Gonçalez de Reyes, a costa de Manuel Meléndez, 1678, BNE, R/ 22696.-Comedias escogidas de diferentes libros de los más célebres e insignes poetas, Bruselas: Manuel Texera Tartar, 1704, BNE, T 23401. 
Vilagrasa publicaría en Valencia el año 1674, con el título Auto glorioso, festejo sagrado con que el insigne Colegio de la preclara Arte de Notaria celebrò la canonizacion del Señor San Luis Beltran... Era, en fin, un personaje bien conocido en la vida intelectual de la Valencia del tercer cuarto de siglo.

De lo dicho hasta ahora hay que consignar que sólo cinco de las comedias escritas en el primer periodo de la producción moretiana (1637-1654) pasaron a esta Parte de comedias de 1676: El Eneas de Dios o El caballero del sacramento o El blasón de los Moncadas (a. 1648), El licenciado Vidriera (1648), La fingida Arcadia (a. 1652), El parecido (1652) y El caballero (1652). Seis de las otras siete comedias tuvieron una impresión anterior a la de la Parte de 1676, pero en todo caso posterior a la Primera parte de 1654 y fueron las tituladas: El valiente justiciero o El ricohombre de Alcalá (1657), No puede ser el guardar una mujer (1659), La fuerza del natural (1662), Primero es la honra (1662), El lindo don Diego (1662), Industrias contra finezas (1666) y Santa Rosa del Perú (1671). El parecido es la única de la que no tenemos constancia que estuviera impresa antes de la publicación de este volumen, aunque todo parece presagiarlo si se tiene en cuenta su texto manuscrito con primeras censuras y aprobaciones de enero de $1652 .{ }^{18}$

Once de esas doce comedias que constituyeron la Segunda parte habían sido, pues, publicadas con anterioridad en varios volúmenes, principalmente de la colección de Comedias escogidas, los cuales se preciaron de llevar cada una de ellos en su índice al menos una pieza de Moreto.

\section{La producción de Moreto del segundo periodo (1655-1669), ajena a la Segunda parte de comedias}

Este segundo periodo de la producción teatral de Moreto (1655-1669) fue también de gran fecundidad. Además de las seis obras ya citadas que se imprimieron en colecciones de Escogidas de ese segundo periodo y pasaron en 1676 al volumen de la Segunda parte de comedias, quedó inacabada Santa Rosa del Perú, que terminó Lanini, como se ha dicho. Había escrito, además, otras catorce comedias, entre las que algunas están pendientes aún de aclarar la atribución:

18. Este mismo manuscrito tiene nuevas censuras de octubre de 1669 y de febrero de 1683 . BNE, Ms. 16423. 


\begin{tabular}{|l|l|}
\hline \multicolumn{1}{|c|}{$\begin{array}{c}\text { Fecha aproximada } \\
\text { de composición de comedias }\end{array}$} & \multicolumn{1}{c|}{ Título } \\
\hline 1656 & $\begin{array}{l}\text { Antes morir que pecar o San Casimiro (atribución } \\
\text { dudosa) }\end{array}$ \\
\hline 1657 & La vida de San Alejo \\
\hline 1657 & El más ilustre francés, San Bernardo \\
\hline 1657 & Fingir y amar \\
\hline 1657 & Amor y obligación \\
\hline ca. 1657 & El Santo Cristo de Cabrillas o El Cristo de los milagros \\
\hline 1662 & Las travesuras de Pantoja \\
\hline a.1668 & La negra por el honor \\
\hline 1668 & Cómo se vengan los nobles \\
\hline a. octubre 1669 & El defensor de su agravio \\
\hline a. octubre 1669 & El ermitaño galán y mesonero del cielo \\
\hline a. octubre 1669 & Yo por vos y vos por otro \\
\hline a. octubre 1669 & $\begin{array}{l}\text { El azote de su patria y renegado Abdenaga o El esclavo } \\
\text { de su hijo (atribución dudosa) }\end{array}$ \\
\hline a. octubre 1669 & $\begin{array}{l}\text { Los hermanos encontrados o Satisfacer callando } \\
\text { (atribución dudosa) }\end{array}$ \\
\hline
\end{tabular}

Por otra parte, antes de 1662 escribió con Matos la comedia hagiográfica San Froilán o El segundo Moisés, pero ya se puede observar que la creación de obras en colaboración con otros dramaturgos decreció de forma notable en este segundo periodo: de 18 comedias en el primer periodo a una sola compuesta de consuno en el segundo. Una de las razones principales, además de los cambios de costumbres, fue la muerte de algunos de sus principales colaboradores, como Belmonte, con el que escribió tres obras y que falleció hacia 1650, o Cáncer, muerto en octubre de 1655, con quien Moreto había compartido la creación de diez comedias, y Martínez de Meneses que fue su colaborador en cinco obras hasta 1661 en que murió. Sólo le sobrevivió Matos Fragoso, con quien escribió la citada San Froilán antes de 1662 y El mejor par de los doce, de fecha insegura. ${ }^{19}$

19. Además de diversas comedias en las que colaboraron otros dramaturgos, por ejemplo,
Cáncer, las cuales pueden, por tanto, adelantarse en su cronología al haber fallecido Cáncer antes. 


\begin{tabular}{|l|l|}
\hline $\begin{array}{c}\text { Fecha aproximada composición de } \\
\text { comedias }\end{array}$ & Título \\
\hline a.1662 & San Froilán o El segundo Moisés (Matos y Moreto) \\
\hline s.d. & El mejor par de los doce (Matos y Moreto) \\
\hline
\end{tabular}

Totales de la producción de Moreto

\begin{tabular}{|l|l|}
\hline $\begin{array}{l}\text { Primer periodo: } 1637-1654 . \\
\text { Obras escritas en solitario }\end{array}$ & 21 comedias \\
\hline $\begin{array}{l}\text { Primer periodo: } 1637-1654 . \\
\text { Obras escritas en colaboración }\end{array}$ & 18 comedias \\
\hline $\begin{array}{l}\text { Segundo periodo: } 1655-1669 . \\
\text { Obras escritas en solitario }\end{array}$ & 21 comedias \\
\hline $\begin{array}{l}\text { Segundo periodo: } 1655-1669 . \\
\text { Obras escritas en colaboración }\end{array}$ & 2 comedias \\
\hline TOTAL & 62 comedias \\
\hline
\end{tabular}

A ellas se sumarían las treinta y cinco piezas breves que han llegado hasta nosotros y que tienen ya edición crítica y estudio preliminar. ${ }^{20}$

\section{El repertorio del segundo periodo de Moreto (1655-1669) en manos de los autores de comedias Diego Osorio, Sebastián de Prado y Juan de la Calle}

En otro lugar me ocupé de la relación de Moreto con algunos de los principales autores de su época, en especial con dos de ellos. ${ }^{21}$ El primero fue Antonio García de Prado, con el que le vinculó una relación que se extendió al menos a cinco años clave en la producción moretiana, los que van de 1645 a 1651, fecha en que falleció Prado. En segundo lugar también observé entonces la relación entre Moreto y Gaspar Fernández de Valdés, el cual cogió el testigo de Prado en 1652 y lo mantuvo hasta 1654 , fecha en que la muerte se lo llevó. Ocho años en total (1646 a 1654), que se convirtieron en un periodo clave si tenemos en cuenta que ése fue precisamente el periodo anterior a la edición de la Primera Parte de Comedias de Moreto (1654), en el que se representó en las tablas la producción ya señalada de aquella etapa, probablemente no muy lejos del momento en que Moreto la compuso. Esos dos nombres: Antonio García de Prado y Gaspar Fernández de Valdés junto al de Diego Osorio, conforman la tríada de autores que

20. Lobato (2003).

21. Lobato $(2008 a)$. 
estuvo vinculada a aquel primer y fructífero periodo de la producción moretiana, que daría lugar a obras del nivel de El desdén, con el desdén.

El segundo periodo de su producción (1655-I669) estuvo marcado por la relación de su obra con los autores Diego Osorio, Sebastián de Prado y Juan de la Calle. La relación con Osorio venía de antiguo. Y él fue también en este segundo periodo el responsable del estreno de varias comedias de Moreto. Sin que sepamos con exactitud de cuál se trata, el lunes 18 de diciembre de 1656 representó «una comedia nueva de Moreto» en el corral de la Cruz de Madrid, en el que su grupo actuó del $15 \mathrm{al} 22$ de aquel mes. ${ }^{22} \mathrm{~A}$ través de la información que nos proporciona Gonzalo de los Ríos, comisario de las comedias de la Villa de Madrid, sabemos que el 25 de enero de 1657 los comediantes de Osorio representaron la comedia San Alejo en el corral del Príncipe y este hecho debió contribuir a que Pedro de la Rosa no tuviera aquel día público en el corral de la Cruz. ${ }^{23}$ Esta comedia de la Vida de San Alejo se repitió en el mismo sitio el 10 de febrero. ${ }^{24}$

Lo cierto es que las fiestas reales habían comenzado una nueva etapa de gran desarrollo a partir de 1655, fecha en que se encargaron al Marqués de Heliche, Jerónimo Montalvo. El baile entremesado El Mellado, dedicado al mundo del hampa, lo escribió para celebrar en 1655 el cumpleaños de la Princesa niña Margarita María, a quien llama "pimpollo tierno» [v. 193]. Ese año se representó también en Madrid la Loa para los años del emperador de Alemania, de Moreto, dedicada a Fernando III, que moriría dos años después, y el dramaturgo se desplazó a Sevilla en 1656, ciudad en la que se representarían algunas de sus piezas breves escritas para el Corpus. Poco posteriores fueron los entremeses El retrato vivo, El hijo del vecino y La reliquia, impresos ya en 1658.

Moreto trabajaba con intensidad en aquellos momentos para palacio, tanto en piezas breves como en otras más extensas. Los entremeses El alcalde de Alcorcón y Las fiestas de palacio se representaron en enero de 1658 con motivo de la salida de la Reina a misa a la Real Capilla y formaron parte de un grupo de festejos con los que el pueblo de Madrid manifestó su contento al ver asegurada la sucesión de la monarquía. Por otra parte, el baile Los oficios coincide en los principales actores con los que representaron el entremés El alcalde de Alcorcón en 1658, por lo que pudieron ponerse en escena en torno a las mismas fechas.

Otros autores compartieron estrenos con Osorio. Fue el caso de Sebastián de Prado y Juan de la Calle que, unidos, actuaban en los corrales madrileños, excepto los días en los que se les reclamaba en palacio. En noviembre de 1659, por ejemplo, representaban en corral la obra de Moreto No puede ser, a la que la documentación llama «nueva comedia» en aquella ocasión, por lo que debió estrenarse entonces. Su puesta en escena en corral no impidió que el 28 y 29 de

22. Varey y Shergold (1973): 221.

23. Varey y Shergold (1973): 82, 222 y 245.

24. Ibidem. 
noviembre se ausentara de allí para ir a palacio. ${ }^{25}$ Estas mismas compañías llevaban en este periodo y hasta el martes de carnaval de 1660 otras dos comedias de Moreto: La misma conciencia acusa y Fingir y amar. ${ }^{26}$ La cohabitación entre corral y corte se dio también en la comedia «vieja» Lo que puede la aprehensión, que el 3 de mayo de 1660 se vio en el corral de la Cruz hecha por la compañía de Jerónimo Vallejo ${ }^{27}$ y al día siguiente la representó Francisca Bezón en palacio.

Volviendo a Diego Osorio, sabemos que hizo en la corte De fuera vendrá un año más tarde, el 28 de noviembre de 1660, esta vez como "comedia vieja» — pues había sido estrenada en 1653 - pero con loa y sainetes nuevos, ${ }^{28}$ llamada a sustituir a La púrpura de la rosa de Calderón, que no estaba aún bien ensayada por su grupo y el de Juana de Cisneros, motivo por el cual no pudo estrenarse hasta el 5 de diciembre, con motivo del tercer cumpleaños del príncipe Felipe Próspero. El 26 de febrero de 1661 los grupos de Osorio y de Juana de Cisneros representaron las piezas breves que acompañaron a la comedia de Moreto Fingir y amar en el Palacio del Buen Retiro, representada ésta por la compañía de Antonio de Escamilla, la cual estaba escrita al menos desde 1659, pues ya se dijo que se había representado también a fines de 1659 o principios de $1660 .{ }^{29}$

Los datos localizados hasta ahora sobre representaciones de comedias permiten suponer que el ritmo de estrenos decreció desde esas fechas hasta los años ochenta en que se repusieron muchas de sus obras, especialmente en palacio, a cargo de las compañías más famosas de la época. Hay que recordar, entre otros avatares, la prohibición de representar comedias en corrales entre los ańos 16651667 con motivo de la muerte del rey, a que luego se hará referencia.

Entre quienes repusieron teatro de Moreto en las tablas en las décadas siguientes, se encuentran autores tan célebres como Manuel Vallejo, Jerónimo García, Francisca Bezón, Simón Aguado, Eufrasia María, Rosendo López de Estrada y Agustín Manuel. Con todo, sí hay datos que permiten afirmar que el dramaturgo continuó escribiendo teatro después de su ordenación sacerdotal en los años sesenta. Poco después de la comedia que hizo en el Buen Retiro, citada antes, preparó para los autos del Corpus de Madrid de 1661 el que sería uno de sus mejores entremeses, El vestuario, dedicado a la vida teatral, y al año siguiente se estrenó su baile El cerco de las hembras, que tiene como tema la crítica de la mujer pidona.

25. Varey y Shergold (1973): 137.

26. Davis y Varey (2003), t. II: 504 . La misma conciencia acusa no era 'nueva', pues se había impreso ya en 1654. Fingir y amar la llevaba en su repertorio Juan Pérez de Tapia en 1657 para representaciones en Andalucía (Llorden, 1975: 195-196). Sin embargo, Davis y Varey dicen que se estrenó en las Carnestolendas de 1660 (Davis y Varey, 2003, t. II: 504). También Juan
Pérez de Tapia llevaba en 1657 para representaciones en Andalucía otras dos comedias de Moreto: El caballero y Amor y obligación (Llorden, 1975: 195-196)

27. Varey y Shergold (1973): 190.

28. Varey y Shergold (1973): 234.

29. En un documento de época se indica que Fingir y amar era obra de tres ingenios (Pérez Pastor, 1914: 464, n 673). 
El cardenal arzobispo de Toledo, don Baltasar de Moscoso y Sandoval, tomó pronto a Moreto bajo su protección. En su biografía se indica que le nombró capellán de la Hermandad del Refugio o de San Pedro. El dramaturgo estuvo en Toledo al menos entre los años 1662 y 1669, en los que era ya sacerdote, vinculado como presbítero a la iglesia de San Nicolás y, frente a opiniones que han defendido que dejo de escribir al ordenarse sacerdote, ${ }^{30}$ el estudio de la cronología de sus obras indica que las compuso y que se representaron hasta su muerte. Por ejemplo, en 1662 se representó el entremés La loa de Juan Rana, para celebrar a la Reina Mariana y también en una fiesta real se hizo El ayo que tuvo como protagonista de nuevo a Juan Rana, esta vez en una de sus últimas actuaciones.

Escribió el entremés La burla de Pantoja y el doctor a partir de uno de los pasajes más celebrados de su comedia Las travesuras de Pantoja, impresa por primera vez en la Parte XIX de Varios, de 1662. El mismo año vio también la publicación de dos de sus grandes comedias: El lindo Don Diego y Primero es la honra. No muy lejos de ellas debió escribirse el entremés El aguador, impreso por primera vez en 1661, que contiene un tema muy relacionado con el de $E l$ lindo, como ya estudié en otro lugar. ${ }^{31}$ Los entremeses Los galanes y La bota debieron ser poco posteriores, pues su primera edición fue en el volumen titulado Tardes apacibles de 1663. En el año 1664 el rey asistió a la representación de La [fingida] Arcadia, realizada en colaboración entre Moreto, un dramaturgo menor y Calderón. Ese mismo año se representó su baile dramático titulado $L a$ Zalamandrana, hermana y se imprimió el entremés Los órganos.

Los corrales del Príncipe y de la Cruz interrumpieron sus representaciones por la muerte del rey Felipe IV entre 1665 y 1667, época precisamente anterior al fallecimiento de Moreto en octubre de 1669. Buena parte de esa década la pasó Moreto en Toledo, como se dijo, y en su testamento de aquel ańo afirmó ser vecino de esa ciudad, a cuyos pobres dejó todos sus bienes. Murió tres días más tarde y no fue enterrado donde él quería, en el cementerio de los pobres, el Pradillo del Carmen, sino en la Capilla de la Escuela de Cristo, en la Parroquia de San Juan. Como es sabido, en aquella época escribía Santa Rosa de Perú, obra que debió terminar su colega en el oficio, Lanini.

De las noticias hasta aquí expuestas podemos concluir que, contra todo pronóstico, la década de los sesenta, tras su ordenación sacerdotal, fue una buena época para su producción dramática, en la que compuso principalmente comedias en solitario y piezas teatrales breves, de las que se recogen las comedias en la tabla que sigue:

30. Nicolás Antonio, 1783: 177

31. Lobato (2008b). 
Incluidas en la Segunda parte de comedias de Agustín Moreto (i 676)

\begin{tabular}{|l|l|}
\hline 1661 palatina & La fuerza del natural (Cáncer, Moreto y Matos) \\
\hline 1662 palatina & Primero es la honra \\
\hline 1662 de figurón & El lindo don Diego \\
\hline 1666 palatina & Industrias contra finezas \\
\hline ca.1669 hagiográfica & Santa Rosa del Perú (Moreto y Lanini) \\
\hline
\end{tabular}

No incluidas en la Segunda parte de comedias de Agustín Moreto (i 676 )

\begin{tabular}{|l|l|}
\hline 1662 & Las travesuras de Pantoja \\
\hline a.1668 & La negra por el honor \\
\hline 1668 & Cómo se vengan los nobles \\
\hline a. octubre 1669 & El ermitaño galán y mesonero del cielo \\
\hline a. octubre 1669 & El defensor de su agravio \\
\hline a. octubre 1669 & Yo por vos y vos por otro \\
\hline a. octubre 1669 & $\begin{array}{l}\text { El azote de su patria y renegado Abdenaga o El } \\
\text { esclavo de su hijo (atribución dudosa) }\end{array}$ \\
\hline a. octubre 1669 & $\begin{array}{l}\text { Los hermanos encontrados o Satisfacer callando } \\
\text { (atribución dudosa) }\end{array}$ \\
\hline
\end{tabular}

La extensión de su corpus teatral es todavía insegura, a pesar de los esfuerzos que se han hecho y se están haciendo en la actualidad. Nuestra contribución a la cronología y fijación de su obra tiene de original el vincularla a las compañías de representantes que la llevaron a escena y el tener en cuenta datos internos de las propias obras, algunos de ellos no atendidos hasta el momento, que ayudan a fijar su fecha de composición o de representación.

El éxito de su producción en su siglo y en el siguiente es innegable, como prueba - entre otros aspectos- el hecho de que sus obras se encuentran en los elencos de las más representadas y que sólo Calderón tenga mayor número de obras compiladas que Moreto en los cuarenta y siete volúmenes de la colección Comedias escogidas impresos durante el siglo XVII. 


\section{Bibliografía general}

Antonio, Nicolás, Biblioteca Hispana Nova sive Hispanorum Scriptorum qui ab anno MD ad MDCLXXXIV floruere... Tomus primus, Matriti, Apud Joachimum de Ibarra Typographum regium, 1783, $2 \mathrm{v}$.

Barrionuevo, Jerónimo de, Avisos de don Jerónimo de Barrionuevo (1654-1658), ed. Antonio Paz y Melia, Madrid, Atlas, colección BAE, 1968, vol. I.

Casaneo, Bartolomé, Catalogus gloriae mundi, laudes, honores, excellentias ac praeminentias omnium fere statuum plurimarumque rerum illius continens, Lugd., Dionysium de Harsy, 1529.

Cassol, Alessandro, «El ingenio compartido. Panorama de las comedias colaboradas de Moreto", Moretiana. Adversa y próspera fortuna de Agustín Moreto, eds. María Luisa Lobato y Juan Antonio Martínez Berbel, Madrid-Frankfurt, Iberoamericana-Vervuert, 2008, pp. 165-184.

Davis, Charles y John E. VAREY, Actividad teatral en la región de Madrid según los protocolos de Juan García de Albertos, 1634-1660. Estudio y documentos, London, Tamesis Books, Fuentes para la Historia del Teatro en España, XXXVXXXVI, 2003, t. II.

Lobato, María Luisa, Loas, entremeses y bailes de Agustín Moreto, Kassel, Reichenberger, 2003, 2 vols.

Loвato, María Luisa, «Moreto, dramaturgo y empresario de teatro. Acerca de la composición y edición de algunas de sus comedias (1637-1654)", Moretiana. Adversa y próspera fortuna de Agustín Moreto, eds. María Luisa Lobato y Juan Antonio Martínez Berbel, Madrid-Frankfurt, Iberoamericana-Vervuert, 2008a, pp. 15-37.

Lовато, María Luisa, "Graciosas tomajonas», La criada en el teatro español del Siglo de Oro, ed. Luciano García Lorenzo, Madrid, Fundamentos, 2008b, pp. 167-182.

Lobato, María Luisa, "Los fundamentos del teatro de Moreto", El teatro del Siglo de Oro. Edición e interpretación, eds. Alberto Blecua, Ignacio Arellano y Guillermo Serés, Madrid-Frankfurt, Universidad de Navarra-Iberoamericana-Vervuert, 2009, pp. 207-229.

Llorden, Andrés, "Compañías de comedias en Málaga (1572-1800)», Gibralfaro. Revista del Instituto de Estudios Malagueños, 27 (1975) pp. 169-200.

Moreto, Agustín, Comedias de Agustín Moreto. Primera parte de comedias, dir. María Luisa Lobato, Presentación; La Primera parte. De la escena al libro, Miguel Zugasti; Preliminares, ed. de Miguel Zugasti; La fuerza de la ley, ed. de Esther Borrego; El mejor amigo, el rey, ed. de Beata Baczynska; El desdén, con el desdén (pp. 397-580), ed. de María Luisa Lobato, Kassel, Reichenberger, Colección Ediciones Críticas 165, 2008, vol. I.

Moreto, Agustín, Comedias de Agustín Moreto. Primera parte de comedias, dir. María Luisa Lobato, coord. Javier Rubiera; Los jueces de Castilla, ed. Abraham 
Madroñal y Francisco Sáez Raposo; El lego del Carmen, San Franco de Sena, ed. Marco Pannarale; Lo que puede la aprehensión, ed. Francisco Domínguez Matito, Kassel, Reichenberger, Colección Ediciones Críticas 171, 2010, vol. IV.

Moreto, Agustín, Comedias de Agustín Moreto. Primera parte de comedias, dir. María Luisa Lobato, coord. Judith Farré; La misma conciencia acusa, ed. Elena Di Pinto y Tania De Miguel; De fuera vendrá, ed. Delia Gavela; Hasta el fin nadie es dichoso, ed. Judith Farré, Kassel, Reichenberger, Colección Ediciones Críticas, en prensa, vol. II.

Nebrisensis, Aelii Antonii, Elementa grammaticae / constructionis \& hispanicis comentariis illustrata per Antonium Cerezum... ,Valentiae, typis Benedicti Macè, vendese en casa de Francisco Duart..., 1675.

Pérez Pastor, Cristóbal, «Nuevos datos acerca del histrionismo español en los siglos XVI y XVII. (Segunda serie. Siglo XVII)», Bulletin Hispanique, 16 (1914) pp. 458-487.

Rennert, Hugo Albert, "Notes on the Chronology of the Spanish Drama", Modern Language Review, 3 (1907-1908) pp. 43-55.

Rull, Enrique, «Procedimientos de construcción triautorial en La fingida Arcadia», De Moretiana Fortuna. Estudios sobre el teatro de Agustín Moreto, Bulletin of Spanish Studies. Hispanic Studies and Researches on Spain, Portugal and Latin America, 85, 7-8 (noviembre 2008) pp. 139-152.

Serrano Morales, José Enrique, Reseña histórica en forma de diccionario de las imprentas que han existido en Valencia desde la introducción del arte tipográfico hasta el año 1868. con noticias bio-bibliográficas de los principales impresores, Valencia, Impr. F. Doménech, 1898-1899.

teatro según Lope de Vega, El, Cuadernos de Teatro Clásico, eds. Pedraza Jiménez, Felipe B., Milagros Rodríguez Cáceres y Mar Zubieta, Madrid, Compañía Nacional de Teatro Clásico 2009, vol. I.

Trambaioli, Marcella, "La fingida Arcadia de 1666: autoría y escritura de consuno", Moretiana. Adversa y próspera fortuna de Agustín Moreto, eds. María Luisa Lobato y Juan Antonio Martínez Berbel, Madrid-Frankfurt, Iberoamericana-Vervuert, 2008, pp. 185-206.

VArey, John E. y Norman E. Shergold, Teatros y comedias en Madrid: 16511665. Estudio y documentos, London, Tamesis Books, Fuentes para la Historia del Teatro en España, IV, 1973.

Vitse, Marc, «El hecho literario», Historia del teatro en España, dir. José María Díez Borque, Madrid, Taurus, 1984, vol. I, pp. 507-612.

Zugasti, Miguel, "Autoridad textual y piratería, con sombras de memorión al fondo, en las dos primeras ediciones de El poder de la amistad (1654), de Moreto", Boletín de la Real Academia Española, en prensa. 